\title{
Feasibility study on color evaluation of jadeite based on GemDialogue color chip images
}

\author{
Ying Guo ${ }^{1^{*}} \mathbb{D}$, Xiang Zong ${ }^{2}$, Ming $\mathrm{Qi}^{3}$, Ye Zhang ${ }^{4}$ and Huan Wang ${ }^{5}$
}

\begin{abstract}
Through the calculation of gemological chromaticity, the green characters and range of chromaticity of 728 cabochon jadeite jade were determined, and then it studies and compares with GemDialogue color card images for jadeite-jade green quality evaluation. It is testified that the method is proved to be effective and the construction of emerald green quality grading system based on it is proved. First of all, with reference to China national standard GB/T 238852009 "Jadeite-jade Grading," the color appearance of jadeite-jade color green was analyzed contrastively with the help of three pieces of GemDialogue color chip images G, Y2G, and B2G where CIE recommended standard light sources D65, A, and CWF, and then it is proposed that the best light source of jadeite-jade color green measuring and evaluating is standard D65 based on quantitative analysis of samples' lightness $L^{*}$, chroma $C^{*}$, hue $h_{0}$. Secondly, according to samples color green and five pieces of GemDialogue color chip images G2Y, Y2G, G, B2G, and G2B, jadeite-jade color was graded in seven levels from the best end "Fancy Vivid" to the last end "Very Light" with the contribution of five different overlap modes of three color masks: black/gray transparent, brown transparent, and black/white opaque. Furthermore, the high accuracy of the method is verified by clustering analysis and Fisher's discrimination reversely. It draws conclusions that there are two evaluation methods of jadeite-jade color green; the first is the colorimetric way of GemDialogue color chip images, and the complementary is the way of Fisher functional method which sample parameters could be back substituted, and all these two methods could be extended to other green gem quality evaluation.
\end{abstract}

Keywords: Jadeite-jade color green, GemDialogue, Color chips, Color zone, Color mask

\section{Introduction}

As the king of all kinds of jade, jadeite formed through metamorphic crystallization and recrystallization from its originals which rich are in $\mathrm{Na}$ and $\mathrm{Al}$, where under high pressure with relatively low temperature. The crystal field theory shows that the color mechanism of jadeite is mainly composed of the following two forms: (1) Metamorphic differentiation: In the even jadeite chemical composition, which in metamorphic circumstance of special temperature, pressure, and flow condition has actively migrated, the color-causing trace elements such as $\mathrm{Cr}^{3+}$ and $\mathrm{Fe}^{3+}$ migrated and aggregated, which then replaced $\mathrm{Al}^{3+}$ in the crystal of jadeite $\mathrm{NaAlSi}_{2} \mathrm{O}_{6}$, and finally, jadeite

\footnotetext{
* Correspondence: yingguocugb@126.com

${ }^{1}$ School of Gemmology, China University of Geosciences (Beijing), Beijing

100083, China

Full list of author information is available at the end of the article
}

reveals color green [1, 2]. (2) Ductile deformation: By the tectonic stress action, jadeite could be ductile deformed under the condition of high pressure and fluid rich with $\mathrm{Cr}^{3+}$ and $\mathrm{Fe}^{3+}$ which is formed by the metasomatism between jadeite and ultrabasic rock that gradually goes into plastic deformation area. Thereby, grains that are formed by dynamic recrystallization, where in the process of ductile deformation, are in the process of nucleation and growth metasomatic displacement; consequently, $\mathrm{Cr}^{3+}$ and $\mathrm{Fe}^{3+}$ go into jadeite crystal lattice and make jadeite green [3].

Because of the complex color-forming process, the shade, lightness, and distribution of jadeite-jade green are not as uniform as a single crystal gem with an optical homogeneity, show the polychromatism caused by the heterogeneity of crystal optics, or reveal the color zonal distribution formed with different grow stages. 
Therefore, based on the calculation of jadeite-jade tristimulus value under different spectral power distribution $[4,5]$, the quality evaluation of jadeite-jade green is influenced not only by its own characteristics but also by its transparency and shape. At the same time, the color of non-spontaneous optical objects is affected by external lighting conditions, such as the color temperature, illuminance, and color rendering of the light source, and the observer, then the color appearance, is not only different with the color temperature [6, 7], there will also be a significant change in illumination [8-11], and it can lead to the international popular research on the color prediction of non-spontaneous optical objects [12-14]. Similar to the color grading system of other materials [15], the color temperature of lighting source must in the scope of $4500-5500 \mathrm{~K}$ and color rendering index must not be lower than 90, which is claimed in the China National Standards (CNS) GB/T 23885-2009 of Jadeite-Jade Grading [16]. Of course, the complexity of on-spontaneous light objects' color appearance under heterogeneous illumination reveals that an inversion relationship is clarified between lightness and brightness and also between chroma and colorfulness under nonuniform illumination with different illuminance levels (high and low) within the same visual field [17]; this is impossible under uniform lighting condition.

The color green has the highest resolution to the human eye, so the change of lighting condition has obvious influence on the appearance of it. Thus, first of all, it should take into account the influencing factors of lighting source while quality evaluation is carried on the color green of jadeite jade, and then the difference in color between the simulated daylight and the various standard light sources with fluorescence were also addressed [18], to evaluate light sources that are suitable for color comparison, i.e., quality evaluation.

Based on the determination of standard lighting sources and observers, the quality evaluation of jadeite-jade green can be compared and evaluated according to the GemDialogue color grading system of the physical color card commonly used in gemstone appreciation of AGTA in the USA. As a portable color card system, it can provide more than 60,000 color samples, and GemDialogue consists of 21 transparent color chips and 3 color masks that cover the major colors of colored gems; each color chip has 10 color bands from high to low; the color values correspond to the number $100,90,80, \ldots . .10$, respectively; and there are 10 bands on each of all 3 masks which is a transparent black/gray, a transparent brown, and an opaque black/ white, also according to the grayscale or color from high to low, which correspond to the number $100,90,80, \ldots \ldots$. 10 , respectively, and used to describe the intensity of black/gray or brown that is hidden in color [19].

\section{Methods}

Firstly, select the green-related color chips, examine the color index of each color band on each color chip, and define the theoretical range of the color green; secondly, jadeite-jade green samples were selected to confirm the actual range of the color green; furthermore, it is smaller than the theoretical range of color green and related colors; therefore, through the actual range of jadeite-jade green, further accurate locating of the range of green band should be taken.

Seven hundred twenty-eight pieces polished cabochon jadeite jade with smooth surface, fine texture, and well purity were selected as samples; all these gemstones are from $6 \mathrm{~mm} \times 7 \mathrm{~mm} \times 5 \mathrm{~mm}$ to $10 \mathrm{~mm} \times 12 \mathrm{~mm} \times 8 \mathrm{~mm}$ which below the upper limit of China National Standard $50 \mathrm{~mm} \times 30 \mathrm{~mm} \times 50 \mathrm{~mm}$, and they display even color within the range from bluish green to vivid green to yellowish green, with continuous changes of color shade and depth.

\section{Samples and experiments}

\subsection{Quantitative analysis of color green}

In view of the non-spontaneous optical object color testing range without fluorescent lighting, it is recommended in $360-830 \mathrm{~nm}$ by CIE (International Illumination Committee), so, to ensure more accurate results, the visible light test range of from $360 \mathrm{~nm}$ to $750 \mathrm{~nm}$ was selected for test of jadeite-jade color green [20].

Based on the uniform color space of CIE 1976 L"a"b", spectrophotometer Color i5 was used to collect reflective signals from Jadeite-Jade surface via the integrating sphere. Test conditions are as follows: specular component setting-excluded (SCE), CIE recommended lighting source D65, spectral range 360 to $750 \mathrm{~nm}$, measurement time $<2.5 \mathrm{~s}$ (flash \& data acquisition), wavelength interval $10 \mathrm{~nm}$, voltage $240 \mathrm{~V}$, and frequency $50 \sim 60 \mathrm{~Hz}$.

All 728 pieces of Jadeite-Jade color green are plotted in the uniform color space CIE $1976 \mathrm{~L}^{\prime \prime} \mathrm{a} " \mathrm{~b}^{\prime \prime}$ [21]; it is illustrated that most color of Jadeite-Jade are concentrated in the area of yellowish green with medium-low lightness, but even no blue tone; and the results are shown in Fig. 1.

X-Rite SP60 portable spherical spectrophotometer is used for color parameter testing, and the testing principle follows the method of testing color in national standard jadeite-jade grading [22-24]. Test conditions are as follows: standard lighting sources which are recommended by the CIE international lighting sources D65, A, and F2; reflection sampling mode that contains mirror reflection settings (SCI Mode); $2^{\circ}$ standard test visual field; measuring range is from 360 to $750 \mathrm{~nm}$; measuring time is less than $2.5 \mathrm{~s}$; wavelength interval is $10 \mathrm{~nm}$; voltage is $240 \mathrm{~V}$; and electric current is $50 \mathrm{~Hz}$. The color tristimulus value of the sample is measured, in order to quantitatively 


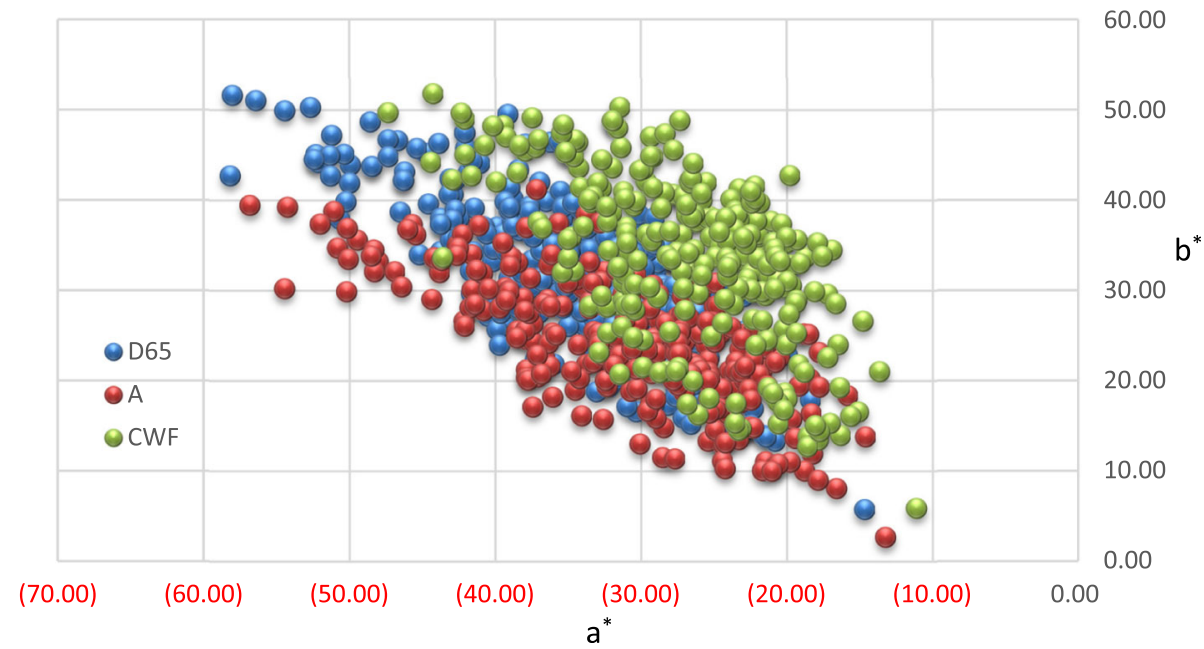

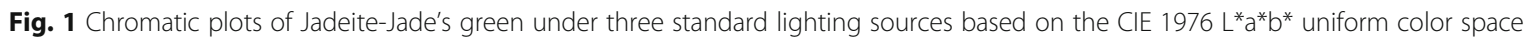

characterize the color, through CIE 1976 L"a"b" uniform color space conversion and then take Munsell color system as color comparison system.

The tristimulus values were converted into the calculation formula of CIE $1976 \mathrm{~L}^{\text {"a" }}$ "b" uniform color spatial coordinates.

$$
\begin{aligned}
& L^{*}=116(Y / Y n) 1 / 3-16 \\
& a^{*}=500[(X / X n) 1 / 3-(Y / Y n) 1 / 3] \\
& b^{*}=200[(Y / Y n) 1 / 3-(Z / Z n) 1 / 3]
\end{aligned}
$$$$
Y / Y n>0.01
$$

where $X, Y$, and $Z$ are the tristimulus values of the test sample; $X \mathrm{n}, Y \mathrm{n}$, and $Z \mathrm{n}$ are the tristimulus values of CIE

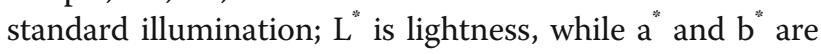
chrominance.

\subsection{Selection of colorimetric lighting sources}

China's national standard GB/T 23885-2009 Jadeite-Jade Grading provisions that the color green of jadeite-jade involves yellowish green, green, and blueish green; therefore, the research on the color green will include green itself and green with blue and yellow tones to varying degrees.

The green color of jadeite-jade has medium to high lightness and high chroma; the color appearance is sensitive to changes in external factors such as lighting source and illuminance color temperature [25]. For example, the color green of jadeite-jade under the different standard lighting sources D65 and A will show the obvious color difference spectrum phenomenon, which termed as metamerism, while under the lighting of a business window or office room, jadeite-jade green will also be affected by environmental fluorescence, and all of these are very unfavorable to jadeite-jade identification and color green evaluation. As a result, the International
Lighting Committee recommended the standard lighting sources D65 (6504K), A (2856K), and CWF (4150K) to simulate daylight, flame light (incandescent light), and cold white fluorescence (American business show) in turn, in order to explore the characteristics and changes of jadeite-jade color appearance under different standard lighting sources, as shown in Fig. 1.

Because of different spectral power distributions, different lighting sources have direct influence on the color appearance of jadeite-jade green, as shown in Table 1.

There are clear and strict requirements for lighting source and measuring environment [26-29], to not only cape series diamond grading system (5500-7200K), but also to fancy color stones such as ruby grading system, sapphire grading system and jadeite-jade grading system (4500-5500K).

The quality appreciation of jadeite-jade green will complete the transformation from the qualitative and semi-quantitative evaluation based on experience to the objective quantitative evaluation, with the application of computer science and the gradual introduction of color science in gemology.

It can be proved that the visual effect of its color appearance is consistent with the theoretical analysis, through computer simulation of chip G under three standard lighting sources, and that shows the pure and vivid green under the standard light D65, green with a hint of yellow under the standard light CWF, and green is slightly blue under the standard light A. Therefore, the color chip of low lightness changes obviously with the change of lighting source, while the color chip of medium to high lightness changes not obviously, as shown in Table 2. Therefore, considering 
Table 1 Statistical colorimetric data and images of three color chips under three different standard lighting source

\begin{tabular}{|c|c|c|c|c|c|c|c|c|c|c|}
\hline \multicolumn{11}{|c|}{ Statistical Colorimetric Data and images of Three Color Chips Under Three Different Standard Lighting Source } \\
\hline & & $\mathrm{L}_{\mathrm{D} 65}^{*}$ & $\mathrm{C}_{\mathrm{D} 65}^{*}$ & $\mathrm{~h}_{0_{\mathrm{D} 65}}$ & $\mathrm{~L}_{\mathrm{CWF}}^{*}$ & $\mathrm{C}_{\mathrm{CWF}}^{*}$ & $\mathrm{~h}_{0 \mathrm{CWF}}$ & $\mathrm{L}_{\mathrm{A}}^{*}$ & $\mathrm{C}_{\mathrm{A}}^{*}$ & $\mathrm{~h}_{0_{A}}$ \\
\hline \multirow{3}{*}{ G } & Max. & 85.84 & 75.10 & 159.09 & 85.16 & 56.46 & 157.90 & 84.68 & 61.68 & 168.06 \\
\hline & Min. & 54.66 & 20.60 & 149.96 & 49.84 & 16.99 & 142.82 & 49.81 & 15.33 & 154.79 \\
\hline & Ave. & 70.02 & 54.60 & 157.16 & 67.12 & 41.81 & 153.76 & 67.44 & 44.39 & 165.57 \\
\hline \multirow{3}{*}{ Y2G } & Max. & 87.27 & 74.37 & 144.20 & 87.21 & 62.33 & 138.10 & 86.96 & 58.08 & 147.20 \\
\hline & Min. & 58.23 & 25.76 & 125.20 & 56.00 & 24.82 & 116.30 & 55.06 & 20.00 & 114.70 \\
\hline & Ave. & 74.17 & 56.45 & 135.10 & 73.02 & 49.39 & 126.90 & 72.20 & 43.77 & 132.30 \\
\hline \multirow{3}{*}{$\mathrm{B} 2 \mathrm{G}$} & Max. & 84.58 & 72.41 & 191.82 & 83.62 & 56.80 & 199.44 & 82.43 & 70.76 & 203.06 \\
\hline & Min. & 60.10 & 19.63 & 174.98 & 54.45 & 19.63 & 177.36 & 53.89 & 22.80 & 189.07 \\
\hline & Ave. & 71.92 & 52.68 & 180.66 & 68.59 & 41.45 & 183.66 & 67.05 & 54.97 & 193.38 \\
\hline
\end{tabular}

the unification with visual color perception and the best display effect of jadeite-jade green, the standard lighting source D65 is selected as for its green comparison and evaluation using GemDialogue color card.

\section{Results and discussion}

Based on the determining the lighting source, jadeite jade green grading will be divided by GemDialogue color card comparison method.

\subsection{Color chip classification}

Based on CIE 1976 L"a"b" uniform color space and D65 illuminating, 5 color chips G2Y, Y2G, G, B2G, and G2B are measured and recorded, together with 3 color masks black/gray transparent, brown transparent and black/ white opaque; there are 10 color bands in each color chip. After its body color determined, each color band of color chips and color masks matches in turn, and the transparent black/gray mask and transparent brown mask adopts both the way of upper cover and the lower cover, while the opaque black/white mask shall be placed only in

Table 2 Green of 10 color zones of color chip G under 3 standard lighting sources

\begin{tabular}{|c|c|c|c|c|c|c|}
\hline \multicolumn{7}{|c|}{ ANOVA of $\mathrm{L}^{*}, \mathrm{a}^{*}$, and $\mathrm{b}^{*}$ of GemDialogue Color Zones } \\
\hline & \multicolumn{2}{|c|}{ Clustering } & \multicolumn{2}{|c|}{ Error } & \multirow{2}{*}{$\mathrm{F}$} & \multirow{2}{*}{ Sig. } \\
\hline & Mean Square & $\mathrm{df}$ & Mean Square & $\mathrm{df}$ & & \\
\hline $\mathrm{L}^{*}$ & 19697.436 & 6 & 34.113 & 1428 & 577.410 & 0.000 \\
\hline$a^{*}$ & 38443.565 & 6 & 30.613 & 1428 & 1255.790 & 0.000 \\
\hline$b^{*}$ & 13716.486 & 6 & 30.894 & 1428 & 443.986 & 0.000 \\
\hline
\end{tabular}


the lower layer, in total, there are 5 ways to overlap color chips. The chromaticity coordinates a", b", and 3 color sensitive parameters $\mathrm{L}^{\prime \prime}, \mathrm{C}^{\prime \prime}$, and $\mathrm{h}_{0}$ significantly corresponded to lightness, chroma, and hue respectively.

The total number of bands measured for each color chip is 510 of each 2550 sets of data, which include both color chip body colors and stack-matched in five modes of the three color masks; in addition, each data set contains the original record data of five color indexes of L", $\mathrm{a}^{\prime \prime}, \mathrm{b}, \mathrm{C}$ ", and $\mathrm{h}_{0}$ (the number before data filtering). To reduce the error, three consecutive in situ measurements were performed in each group, and then its arithmetic mean is taken into account.

In China's National Standard Jadeite-Jade Grading, according to the main wavelength value of spectral colors, the color of jadeite-jade green is divided into green (G) with $500 \leq \lambda<530$, yellowish green (yG) with $530 \leq \lambda<550$, and bluish green (bG) with $490 \leq \lambda<500$ [8]. Considering the large number of previous research findings, the main wavelength range of the spectral color of green jadeite-jade is greater than the range of $490 \leq \lambda<550$ set by the national standard for jadeitejade grading. After converting to hue, $\mathrm{h}_{0} \in(120,180)$ was selected to divide colors according to the range of hue angles measured in this study. A total of 3536 groups of data were screened out, after much more deviant color difference from jadeite-jade stone to measured data weed out, for example, yellowish green, and among them, there are 1435 pure green bands that accord with the hue range. The green band which selected out has a wide range of lightness and even transition, with a big lightness range $\mathrm{L}^{\prime \prime} \in(28.18,87.27)$ and chroma range $C^{\prime \prime} \in(3.21,75.10)$. It represents the overall distribution of jadeite-jade green in uniform color space and satisfies the comparison and classification of the color green.

K-means clustering analysis is adopted to try to classify all data into $5,6,7,8$, and 9 categories. The concomitant probability of the $F$ statistics of L", a", and $\mathrm{b}^{\prime \prime}$ in the three-dimensional coordinate of color is all less than 0.01 , indicating that there is a significant difference between different categories. With reference to the Gemological Institute American (GIA) color diamond color nine categories of odd classification evaluation system, and considering the simplified categories as far as possible for practical reasons, the total number of effective GemDialogue will evaluate the emerald green ribbon classified into 7 classes, as shown in Table 3.

The principle of classification is based on the distance between sample variables and established class centers, and the computer simulation of representative color blocks is shown in Table 4.

It can be seen from Table 4 that the color of the third category of color blocks is the best with medium-high
Table 3 ANOVA of $L^{*}, a^{*}$, and $b^{*}$ of GemDialogue color zones

\begin{tabular}{|c|c|c|c|c|c|c|}
\hline & Clustering & & Error & & $F$ & Sig. \\
\hline & Mean square & df & Mean square & $\mathrm{df}$ & & \\
\hline $\mathrm{L}^{*}$ & $19,697.436$ & 6 & 34.113 & 1428 & 577.410 & 0.000 \\
\hline$a^{*}$ & $38,443.565$ & 6 & 30.613 & 1428 & 1255.790 & 0.000 \\
\hline$b^{*}$ & $13,716.486$ & 6 & 30.894 & 1428 & 443.986 & 0.000 \\
\hline
\end{tabular}

lightness $\mathrm{L}^{*}$ and highest chroma $\mathrm{C}^{*}$, which is characterized by the highest absolute value of $\mathrm{a}^{\prime \prime}$, and indicated the lowest absolute value of $b^{\prime \prime}$, which deviates from green; that is, all parameters point to the most brilliant green, so it is classified as the highest quality level of vivid fancy green: marked as "Fancy Vivid", represents 135 color blocks, accounting for $9.41 \%$ of total color blocks. The first category of color block quality is slightly lower, even though the lightness is slightly higher than the level before the third class, but the chromaticity of the medium a" and b" that makes its chroma is slightly lower than the third class and obviously biased towards the slight yellow tone, with color appearance shows Yang green, so it is classified as a high-level of intense fancy green, marked as "Fancy Intense," represents 129 color blocks, accounting for 8.99\% of total color blocks. The color of the sixth category of color block is similar to that of the former category with similar medium chroma a" and lower b", it performed weakening yellow tone and reducing chroma which its color appearance shows medium green, so it is classified as deep fancy green, marked as "Fancy Deep", represents 159 color blocks, accounting for $11.08 \%$ of total color blocks. The lightness of the second category of color blocks continues to decrease. Although the color is similar to the former category 6 , the color appearance is dark green, so it is classified as dark fancy green: marked as "Fancy Dark", represents 126 color blocks, accounting for $15.05 \%$ of total color blocks. The lightness of color of category 5 is medium, and the color is some lower than that of the former category 2. The appearance of color is pale dark green, so it is classified as fancy green: marked as "Fancy", represents 280 color blocks, accounting for $19.51 \%$ of total color blocks. Although the color of the fourth kind of color has relatively high lightness, its chroma is lower than the previous grade of the fifth kind, and its color appearance is pale green, so it is classified as light fancy green: marked as "Fancy Light", represents 186 color blocks, accounting for $12.96 \%$ of total color blocks. The lightness and chorma of the seventh color block are the lowest among all the seven colors categories. The color appearance is pale green with obvious gray tone. For the green tone, the color is very light, so it is classified as very light green: marked as "Very Light," represents 186 color blocks, accounting for $12.96 \%$ of total color blocks. Through cluster analysis, the boundary between the seven categories of 1435 test blocks is clear, the proportion of 
Table 4 Computer simulation images of Gemdialogue color blocks of 7 cluster centers of jadeite-jade green

\begin{tabular}{|c|c|c|c|c|}
\hline & Cluster Center & Appr. & Computer simulation of GemDialogue color blocks of 7 clusters & Grade \\
\hline 1 & $\begin{array}{l}\mathrm{L}^{*}=58.00 \\
\mathrm{a}^{*}=-35.48 \\
\mathrm{~b}^{*}=32.26\end{array}$ & $\begin{array}{l}\text { Fancy } \\
\text { Intense } \\
(129)\end{array}$ & & II \\
\hline 2 & $\begin{array}{l}\mathrm{L}^{*}=43.58 \\
\mathrm{a}^{*}=-31.20 \\
\mathrm{~b}^{*}=5.68\end{array}$ & $\begin{array}{l}\text { Fancy } \\
\text { Dark } \\
(216)\end{array}$ & & IV \\
\hline 3 & $\begin{array}{l}\mathrm{L}^{*}=53.60 \\
\mathrm{a}^{*}=-52.60 \\
\mathrm{~b}^{*}=10.54\end{array}$ & $\begin{array}{l}\text { Fancy } \\
\text { Vivid } \\
(135)\end{array}$ & & I \\
\hline 4 & $\begin{array}{l}\mathrm{L}^{*}=64.42 \\
\mathrm{a}^{*}=-17.02 \\
\mathrm{~b}^{*}=11.43\end{array}$ & $\begin{array}{l}\text { Fancy } \\
\text { Light } \\
(186)\end{array}$ & & VI \\
\hline 5 & $\begin{array}{l}\mathrm{L}^{*}=45.65 \\
\mathrm{a}^{*}=-21.04 \\
\mathrm{~b}^{*}=16.86\end{array}$ & $\begin{array}{l}\text { Fancy } \\
(280)\end{array}$ & & $\mathrm{V}$ \\
\hline 6 & $\begin{array}{l}\mathrm{L}^{*}=58.39 \\
\mathrm{a}^{*}=-34.83 \\
\mathrm{~b}^{*}=12.42\end{array}$ & $\begin{array}{l}\text { Fancy } \\
\text { Deep } \\
(159)\end{array}$ & & III \\
\hline 7 & $\begin{array}{l}\mathrm{L}^{*}=38.36 \\
\mathrm{a}^{*}=-10.04 \\
\mathrm{~b}^{*}=5.54\end{array}$ & $\begin{array}{l}\text { Very } \\
\text { Light } \\
(330)\end{array}$ & & VII \\
\hline
\end{tabular}

each class is in accordance with the normal distribution, and the consistency among individual cases in the class is very high.

\subsection{Verification of color chip classification results}

Based on clustering analysis, the establishment of the dependent variable categories and the independent variable discriminant function between the $\mathrm{L}^{*}, \mathrm{a}^{*}$, and $b^{*}$, through a reverse authentication, for example, has been divided into categories of sample is divided into the categories of inspection right, for which testifies the validity and effectiveness on the entire range of color band color grading. At the same time, GemDialogue color green chips of the test piece is only basic covers the jadeite-jade green, but because of the complex causes, jadeite-jade riches in colors, although numerous test data, it has only certain representativeness in the color green range, not completely contain all of the jadeite-jade green in nature. Therefore, through the discriminant function established by the discriminant analysis, the jadeite-jade green color correlation color outside the analysis sample is substituted into the discriminant function to simulate the graded colors.

The discriminant function was established by using the Fisher discriminant method to analyze the 7 categories. The independent variable coefficient and the function constant of the discriminant function are shown in Table 5.

$L^{*}, a^{*}$, and $b^{*}$, the sample parameters to be verified and classified, was substituted into 7 Fisher function expressions. By comparing the function values of each function, it was shown that the observed value belongs

Table 5 Fisher discriminant coefficient and constant of Gemdialogue color blocks of imitated jadeite-jade green

\begin{tabular}{llllllll}
\hline & 1 & 2 & 3 & 4 & 5 & 6 & 7 \\
& Fancy intense & Fancy dark & Fancy vivid & Fancy light & Fancy & Fancy deep & Very light \\
\hline$L^{*}$ & 1.525 & 1.170 & 1.374 & 1.840 & 1.248 & 1.585 & 1.099 \\
$a^{*}$ & -0.835 & -0.856 & -1.519 & -0.270 & -0.459 & -0.892 & -0.163 \\
$b^{*}$ & 0.792 & -0.038 & 0.014 & 0.162 & 0.369 & 0.137 & 0.055 \\
Constant & -73.779 & -40.689 & -78.794 & -64.432 & -38.369 & -64.607 & -23.996 \\
\hline
\end{tabular}


to this category. The results of classification discriminant analysis of 1435 samples are shown in Table 6 .

Through the classification result matrix, it can be seen that only 29 samples not in accordance with the actual classification, accounting for $2.02 \%$ of the total, with satisfactory accuracy. The accuracy of the discriminant analysis of samples divided into categories was as low as 96.3\% of the second category (Fancy Dark), as high as $100.0 \%$ of the third category (Fancy Vivid), and as high as $98.0 \%$ of the overall accuracy. Precision discriminant analysis results show that the effect is ideal, and based on a large number of sample data clustering analysis, the function formula of Fisher discriminant analysis is feasible. So, the accuracy and simplicity of Fisher's discriminant function formula can be used to evaluate the quality of physical green jadeite.

\subsection{The application and feasibility of color chip in jadeite- jade green evaluation}

The premise of appreciating jadeite-jade color green with GemDialogue color chips is as follows: (1) it is recommended to use standard lighting source D65 or similar northern clear sky natural light at $10 \mathrm{am}$; (2) visual inspection of cleaning samples and color chips; (3) 5 chips G2Y, Y2G, G, B2G, and G2B should be used and with 3 color masks black/gray transparent, brown transparent and black/white opaque. Because all the color band chroma of upper-stack mask is lower than lowerstack mask obviously, it is recommended to adopt the lower-stack mask [19]. Therefore, when measuring a green jadeite-jade with gray black or brown tone, it is necessary to record the position of lower-stack mask

Table 6 Fisher discrimination count and accuracy of GemDialogue color blocks of imitated Jadeite-Jade green

\begin{tabular}{|c|c|c|c|c|c|c|c|c|c|}
\hline & & 1 & 2 & 3 & 4 & 5 & 6 & 7 & \\
\hline \multirow{7}{*}{$\begin{array}{l}\text { Discri. } \\
\text { count }\end{array}$} & 1 & 127 & 0 & 0 & 2 & 0 & 0 & 0 & 129 \\
\hline & 2 & 0 & 208 & 2 & 1 & 0 & 5 & 0 & 216 \\
\hline & 3 & 0 & 0 & 135 & 0 & 0 & 0 & 0 & 135 \\
\hline & 4 & 0 & 0 & 0 & 184 & 0 & 2 & 0 & 186 \\
\hline & 5 & 0 & 1 & 0 & 1 & 275 & 0 & 3 & 280 \\
\hline & 6 & 0 & 0 & 0 & 3 & 1 & 155 & 0 & 159 \\
\hline & 7 & 0 & 4 & 0 & 1 & 3 & 0 & 322 & 330 \\
\hline \multirow{7}{*}{$\begin{array}{l}\text { Discri. } \\
\text { accuracy }\end{array}$} & 1 & 98.4 & 0 & 0 & 1.6 & 0 & 0 & 0 & 100.0 \\
\hline & 2 & 0 & 96.3 & 0.9 & 0.5 & 0 & 2.3 & 0 & 100.0 \\
\hline & 3 & 0 & 0 & 100.0 & 0 & 0 & 0 & 0 & 100.0 \\
\hline & 4 & 0 & 0 & 0 & 98.9 & 0 & 1.1 & 0 & 100.0 \\
\hline & 5 & 0 & 0.4 & 0 & 0.4 & 98.2 & 0 & 1.1 & 100.0 \\
\hline & 6 & 0 & 0 & 0 & 1.9 & 6 & 97.5 & 0 & 100.0 \\
\hline & 7 & 0 & 1.2 & 0 & 0.3 & 9 & 0 & 97.6 & 100.0 \\
\hline
\end{tabular}

The overall classification accuracy is $98.0 \%$ mode to correspond certain color bands. The record results were then compared with 1435 measuring bands and the sample color quality was graded according to the test database category. If the colorimetric band is determined to be band 80 of chip G, with band 10 of lower-stack black/gray transparent mask, then the color record is G80/G10\%, which corresponds to category 3, and its qualified as the top grade positive green (Fancy Vivid). If there is no matching data in the existing database, the interpolation method of colorless diamond color quality evaluation is recommended, that is, the color of adjacent bands should be consulted.

As an important supplement, because of the diversity of GemDialogue color chip combination methods, when it is not suitable for direct color comparison and color evaluation with color chips, color parameters of jadeitejade to be evaluated can be measured directly under D65 standard light source, substitute L", a", and b" into the established Fisher discriminant function, then the maximum value of each class function is the category to which it belongs, and the corresponding color grade is determined. Seven categories have strong independence and clear boundaries from each other, but all these is only based on the five color chips in the GemDialogue color system, so the universality of representation will be supplemented by the continuous enrichment of databases, and can be extended to other green gems color evaluation, for example, emerald with vivid green, peridot with clearly yellow tone.

\section{Conclusions}

Based on GemDialogue color system, the application research of jadeite-jade green grade division and quality evaluation is done, and a conclusion is drawn:

1. The parameters which affect jadeite-jade green color were studied by using three standard lighting sources, the results indicates the following: (1) the jadeite-jade green lightness L" under the three lighting sources is similar in general, while that of the light source D65 is slightly higher; (2) chromaticity a" and chroma $C^{*}$ show the highest under light source D65 with the pure green of color appearance and light source CWF casts slight yellow tone on the stones; therefore, it is not suitable for light yellow tone or blue tone jadeite-jade green, and some partial turquoise samples under light source A crisper slightly higher than the D65 light source, the chroma of partial bluegreen samples under light source A is slightly higher than that of light source D65. For comprehensive consideration, light source D65 is recommended as the standard light source for jadeite-jade green measurement or quality evaluation. 
2. Takes L", a", and b" of color band as variables to perform K-Means fast clustering analysis, the 1435 measuring data are graded into seven categories, the concomitant probability of $\mathrm{F}$ statistic between 7 categories was significantly lower than the significance level of 0.01 , it shows the ideal clustering effect. The Fisher discriminant function was established for the 7 categories of clustering, the accuracy of discriminant analysis classification was verified as high as $98 \%$, it shows that the method of fast clustering analysis and discriminant analysis of measuring data is scientific and reasonable, and the accuracy of the discriminant function is also satisfied for the color classification of unknown samples, so it is effective and feasible to apply GemDialogue color card to green jade color quality grade evaluation.

3. Color parameters and color simulation of samples in seven categories have been graded, which corresponding to the division of various color quality grade, and comprehensive analysis was made from the color parameters L", a", and b", and the color quality grades are arranged in order from the best end of Fancy Vivid, then Fancy Intense, Fancy Deep, Fancy Dark, Fancy, Fancy Light, and Very Light in the sequence. It is resulted that the color quality grade determines the commercial value of green to be evaluated.

4. Through the practical application of GemDialogue color card in jadeite-jade green quality evaluation, the GemDialogue color card colorimetric method and the method of replacing the Fisher function with the color parameter of the complementary color comparison sample were determined. The first method can be used for most of the color samples to be compared, and the second method may be used for a small number of samples that do not clearly correspond to the color card database, to ensure that the color quality grade of each color sample can be obtained.

5. Based on GemDialogue color system, to enhance the universality of color quality evaluation through the continuous enrichment of database, it can be extended to other green gems color evaluation, for example, emerald with vivid green, peridot with clearly yellow tone.

\begin{abstract}
Abbreviations
A: Flame light (incandescent light); CIE: International illumination committee; CNS: China National Standards; CWF: Cold white fluorescence (American business show); D65: Daylight; F2: Cool White Fluorescent; GB/ 23885-2009: GuojiaBiaozhun/Tuijian; GIA: Gemological Institute American; SCE: Specular component setting excluded; SCI Mode: Mirror reflection settings
\end{abstract}

\section{Funding}

No funding was received.

\section{Availability of data and materials}

Data will not be shared; reason for not sharing the data and materials is that the work submitted for review is not completed. The research is still ongoing, and those data and materials are still required by the author and co-authors for further investigations.

\section{Authors' contributions}

GY designed the research. $X Z, Q M, Z Y$, and WH analyzed the data. GY wrote and edited the manuscript.

\section{Authors' information}

Guo Ying is a researcher of Mineralogy and Gemmology and an associated professor in School of Gemmology, China University of Geosciences (Beijing). He mainly focuses on data acquisition and storage of colorimetry, data compression, intelligent testing technology, micro and nano sensor, and testing technology. In recent years, he has completed several projects and published 51 research papers.

Xiang Zong is the master of materials engineering in China University of Geosciences (Beijing), a researcher of gemological mineralogy, a certified gemologist of China. During the master's degree, he mainly focuses on the data testing and analysis of the Colorimetry and the color evaluation of gemstone. He is also the manager of Beijing Huaxia Pawnshop Co., Ltd. Qi Ming is the lecturer of the Gemology Teaching and Research Section, Liaoning Geology Engineering Vocational College. She mainly focuses on gemstone identification and market research.

Zhang Ye is a teaching assistant in Dehong Teachers' College, Department of Science and Technology. She mainly focuses on the trade of precious jade stone and the identification of color, texture, and imitation of precious stone Wang Huan is a teacher of Beijing City of Arts and Crafts Senior Technical School. She mainly focuses on data acquisition and storage of colorimetry, data compression, intelligent testing technology, micro and nano sensor, and testing technology. In recent years, she has completed several projects and published two research papers.

Ethics approval and consent to participate

We approved.

Consent for publication

We agreed.

\section{Competing interests}

The authors declare that they have no competing interests.

\section{Publisher's Note}

Springer Nature remains neutral with regard to jurisdictional claims in published maps and institutional affiliations.

\section{Author details}

${ }^{1}$ School of Gemmology, China University of Geosciences (Beijing), Beijing 100083, China. ${ }^{2}$ Huaxia Pawnshop Co., Ltd, Dongcheng District, Beijing 100062, China. ${ }^{3}$ Gemmology Teaching and Research Section, Liaoning Geology Engineering Vocational College, Dandong 118008, Liaoning, China. ${ }^{4}$ Department of Science and Technology, Dehong Teachers' College, Luxi City 678400, Yunnan, China. ${ }^{5}$ Teaching Management Center, Beijing City of Arts and Crafts Senior Technical School, Beijing 102200, China.

Received: 13 July 2018 Accepted: 19 September 2018 Published online: 29 September 2018

\section{References}

1. T.J.B. Holland, R. Powell, An internally-consistent thermodynamic data set for phases of petrological interest. J. Metamorph. Geol. 16, 309-343 (1998)

2. R.W. White, R. Powell, TJ.B. Holland, B.A. Worley, The effect of $\mathrm{TiO} 2$ and Fe2O3 on metapelitic assemblages at greenschist and amphibolite facies conditions: mineral equilibria calculations in the system $\mathrm{K} 2 \mathrm{O}-\mathrm{FeO}-\mathrm{MgO}-\mathrm{Al} 2 \mathrm{O} 3-\mathrm{SiO} 2-\mathrm{H} 2 \mathrm{O}-$ TiO2-Fe2O3. J. Metamorph. Geol. 18, 497-512 (2000) 
3. A. Pasdar, H.H. Mehne, Intelligent three-phase current balancing technique for single-phase load based on smart metering. Electr. Power Energy Syst. 33, 693-698 (2011)

4. A.M. Shams-Nateri, Estimation of CIE tristimulus values under various illuminants. Color. Res. Appl. 34(2), 100-107 (2009)

5. X. Wang, Y. Gong, D. Song, Design and study of a color sensitivity function. Color. Res. Appl. 30(2), 118-124 (2005)

6. E.-M. Yang et al., Dependence of the color appearance of some flowers on illumination. Color. Res. Appl. 39(1), 28-36 (2014)

7. S.-H. Kim, Y.-K. Lee, B.-S. Lim, Metameric effect between dental porcelain and porcelain repairing resin composite. Dent. Mater. 23, 374-379 (2007)

8. H.S. Cha, Y.K. Lee, Difference in illuminant-dependent color changes of shade guide tabs by the shade designation relative to three illuminants. Am. J. Dent. 22(6), 350-356 (2009)

9. A. Daugirdiene, J.J. Kulikowski, IJ. Murray, J.M.F. Kelly, Test illuminant location with respect to the Planckian locus affects chromaticity shifts of real Munsell chips. J. Opt. Soc. Am. A 33(3), A77-A84 (2016)

10. E.C. Fuchs, K. Gatterer, Colour change of co-doped yttrium aluminium borate crystals under illumination with different white light sources. Cent. Eur. J. Chem. 6(4), 497-504 (2008)

11. S. Hong et al., Evaluation of the visibility of colored objects under LED lighting with various correlated color temperatures. Color. Res. Appl. 42(1), 78-88 (2017)

12. M. Sánchez-Marañón, P.A. Garcíab, et al., Influence of natural daylight on soil color description: assessment using a color-appearance model. Soil Sci. Soc. Am. J. 75(3), 984 (2011)

13. K.M. Braun, M.D. Fairchild, Testing five color-appearance models for changes in viewing conditions. Color. Res. Appl. 22(3), 165-173 (1997)

14. Y. Park, M.R. Luo, C.J. Li, Y. Kwak, Refined CIECAMO2 for bright surround conditions. Color. Res. Appl. 40(2), 114-124 (2015)

15. C. Boukouvalas, Color grading of randomly textured ceramic tiles using color histograms. IEEE Trans. Ind. Electron. 46(1), 219-226 (1999)

16. GB/T 23885-2009, Jadeite Grading[P], China National Bureau of Technical Supervision. Chinese National Standards (Standards Press of China, Beijing, 2009)

17. Y. Nayatani, H. Sakai, Complexity of the color appearance of objects with nonuniform illumination. Color. Res. Appl. 32(5), 372-377 (2007)

18. Y. Guo, H. Wang, L. Xiang, S. Dong, Metamerism appreciation of jadeite-jade green under the standard light sources D65, a and CWF. Acta Geologica Sinica 90(6), 2097-2103 (2016)

19. Y. Guo, X. Zong, M. Qi, Feasibility study on quality evaluation of jadeite-jade color green based on GemDialogue color chip. Multimedia Tools Appl., 116 (2018) https://doi.org/10.1007/s11042-018-5753-7

20. Z. Wang et al., Interpolation, extrapolation, and truncation in computations of CIE tristimulus values. Color. Res. Appl. 42(1), 10-18 (2017)

21. C. Sheridan, M. O'Farrell, et al., A comparison of CIE $L^{*} a^{*} b^{*}$ and spectral. methods for the analysis of fading in sliced cured ham. J. Opt. A Pure Appl. Opt. 9(6), 32-39 (2007)

22. GB/T 16554-2010. Diamond Grading[P], China National Bureau of Technical Supervision. Chinese National Standards (Standards Press of China, Beijing, 2009)

23. GB/T 32862-2016. Sapphire Grading[P], China National Bureau of Technical Supervision. Chinese National Standards (Standards Press of China, Beijing, 2009)

24. GB T 32863-2016. Ruby Grading[P], China National Bureau of Technical Supervision. Chinese National Standards (Standards Press of China, Beijing, 2009)

25. S.W. Hsiao, C.J. Tsai, A residual modified transformation formula from Munsell to sRGB color system. Color. Res. Appl. 40(3), 243-255 (2015)

26. B. George, V.I. Morgan, A spreadsheet for calculating normative mole fractions of end-member species for $\mathrm{Na}$-Ca-Li-Fe2+-Mg-Al tourmalines from electron microprobe data. Am. Mineral. 101, 111-119 (2016)

27. Y. Guo, Z. Xinyan, L. Xiang, Z. Ye, Quantitative characterization appreciation of golden citrine golden by the irradiation of [FeO4]4. Arab. J. Chem 11(6), 918-923 (2018)

28. Y. Guo, Quality evaluation of tourmaline red based on uniform color space. Clust. Comput., 20(4), 3393-3408 (2017) https://doi.org/10.1007/s10586-0171091-1

29. Y. Guo, Quality grading system of jadeite-jade green based on three colorimetric parameters under CIE standard light sources D65, CWF and A. Bulg. Chem. Commun. 49(4), 961-968 (2017)

\section{Submit your manuscript to a SpringerOpen ${ }^{\circ}$ journal and benefit from:}

- Convenient online submission

- Rigorous peer review

- Open access: articles freely available online

- High visibility within the field

- Retaining the copyright to your article

Submit your next manuscript at $\boldsymbol{\nabla}$ springeropen.com 